\title{
COMPARACIÓN DEL GRADO DE MICROFILTRACIÓN MARGINAL EN INTERFASE DIENTE/COMPOSITE ENTRE SISTEMAS ADHESIVOS DE GRABADO TOTAL Y AUTOGRABADO.
}

\author{
Comparison of de degree of de marginal microleakage at the composite/thooth \\ interface between total etch and self-etch adhesive systems.
}

\author{
Andrea Córdova López ${ }^{1}$, Paulo Bustos González ${ }^{2}$, Patricio Sarmiento Criollo ${ }^{3}$. \\ ${ }^{1}$ Odontólogo Graduado, Universidad Católica de Cuenca, Cuenca, Ecuador. \\ 2 Odontólogo Graduado, Universidad Católica de Cuenca, Cuenca, Ecuador. \\ ${ }^{3}$ Magíster en Odontología Restauradora y Estética Dental, Académico de la Carrera de Odontología \\ Universidad Católica de Cuenca, Cuenca, Ecuador. \\ *od.andreacordoval@hotmail.com
}

\begin{abstract}
Resumen
Objetivos: comparar el grado de microfiltración en interfase diente/composite entre sistemas adhesivos de grabado total y autograbado. Materiales y métodos: se realizó un estudio experimental in vitro con 62 premolares extraídos, sanos; a los cuales se les realizó una preparación cavitaria estandarizada Clase V, ubicada en la cara vestibular a $1 \mathrm{~mm}$ de la línea amelocementaria, las cuales se restauraron con composite de microrelleno. Se dividió en 2 grupos, el grupo A (sistema adhesivo de grabado total) y el grupo B (sistema adhesivo de autograbado), posteriormente la muestra fue conservada en baño maría a $37^{\circ} \mathrm{C}$ por 24 horas. Se sometió la muestra a termociclado ( 300 ciclos), y se sumergieron en tinción para finalmente ser recortadas sentido longitudinal y observadas en esteroscopio determinando la ausencia o presencia de microfiltración marginal. Resultados: El 84,84\% de especímenes del grupo A (sistema adhesivo de autograbado) presentaron microfiltración marginal; mientras que el $57,57 \%$ de especímenes del grupo B (sistema adhesivo de grabado total) Conclusiones: La microfiltración marginal observada fue alta independiente al sistema adhesivo utilizado.
\end{abstract}

Palabras clave: Microfiltracion, Sistemas Adhesivos, composite dental.

\begin{abstract}
Aim: to compare the degree of microleakage at the tooth/composite interface between total etch and self-etch adhesive systems. Material and methods: an experimental in vitro study was carried out with 62 premolars extracted, healthy; to which a Class $V$ standardized cavitary preparation was made, located on the vestibular face $1 \mathrm{~mm}$ from the amelocementary line, which were restored with micro-filled composite. It is divided into 2 groups, group A (total etching adhesive system) and group B (self-etching adhesive system), subsequently the sample was kept in a water bath at $37^{\circ} C$ for 24 hours. The sample was subjected to thermocycling (300 cycles), and it was immersed in staining to finally be cut longitudinally and observed by stereoscope determining the absence or presence of marginal microleakage. Results: $84.84 \%$ of specifications of group A (self-etching adhesive system) marginal microleakage considerations; while $57.57 \%$ of the examples of group B (total etching adhesive system) Conclusions: The marginal microleakkage observed was high independent of the adhesive system used.
\end{abstract}

Key words: Micro-leakage, adhesive systems, dental composite.

\section{Introducción}

Los sistemas adhesivos son biomateriales que integran la superficie dentaria con la superficie del material restaurador. $^{1}$ Los adhesivos utilizados en odontología restauradora con mayor frecuencia en la actualidad son de dos tipos: I) los de grabado total (Total-Etch Systems), los cuales requieren de un grabado ácido (ácido ortofosfórico) para disminuir el barrillo dentinario previo a su utilización, 
y II) los de autograbado (Self-Etch Systems), que contiene su propio ácido e incorpora el barrillo dentinario a la capa híbrida. ${ }^{2,3}$

La microfiltración marginal es el paso de partículas y microrganismos a la interfase que se forma entre la resina y el diente, causando cambios de coloración, hipersensibilidad postoperatoria, caries recidivante e inflamación pulpar. ${ }^{4} \mathrm{El}$ grado de microfiltración es valorado del 0 al 4, mediante la escala propuesta por Alavi y Kianimanesh en el año 2002.

El uso de diferentes sistemas adhesivos es controversial. Esto se debe, en parte, a que los fabricantes han reducido el número de pasos clínicos para la aplicación de los mismos; lamentablemente, esto se ha logrado a expensas de un aumento de la microfiltración. ${ }^{6,7}$ Se ha demostrado en la mayoría de investigaciones la superioridad de los sistemas adhesivos de grabado total como agentes de unión en comparación con los sistemas de autograbado. Por ejemplo, Vinay et al. ${ }^{8}$ encontraron menores valores de microfiltración en adhesivos autograbantes en comparación con el de grabado total. Sin embargo, otros autores no han encontrado diferencias significativas entre estos adhesivos o incluso han reportado la superioridad del adhesivo autograbante. Tal es el caso del trabajo publicado por Tabari et al. ${ }^{9}$ quienes no encontraron diferencias significativas entre los valores de microfiltración entre los dos sistemas adhesivos evaluados.

Pese a que existe un número limitado de investigaciones a nivel internacional que comparan el grado de microfiltración de estos sistemas adhesivos empleando estrategias metodológicas que simulan cambios bruscos de temperatura de la cavidad oral (termociclado) ${ }^{10}$ este tipo de estudios no se han realizado en nuestro país. Por lo tanto el objetivo de nuestro estudio fue comparar el grado de microfiltración marginal en la interfase diente/resina utilizando sistemas adhesivos autograbantes y de grabado total, empleando la técnica anteriormente mencionada.

\section{Materiales y métodos}

Nuestro estudio tuvo un enfoque de tipo experimental, descriptivo en el cual se examinó una muestra de 66 piezas dentarias (premolares inferiores) completamente sanas sin ninguna afectación en sus tejidos; cada espécimen fue higienizado minuciosamente para remover todo tipo de tejido periodontal y óseo (cureta periodontal $\mathrm{Hu}$-Friedy ${ }^{T M}$ y se conservaron en un frasco estéril con suero fisiológico $0,9 \mathrm{gr}$ para mantenerlos hidratados.

En cada espécimen se preparó una cavidad V de Black en su cara vestibular a 1 milímetro de la línea amelocementaria con una pieza de alta velocidad (NSK PANA MAX SUB 2) misma que fue calibrada para no emitir partículas oleosas, utilizando una fresa de diamante redonda 1012 (KENDO), que fue remplazada cada cinco preparaciones cavitarias, el biselado de la cavidad fue de $0,5 \mathrm{~mm}$, y se utilizó una fresa cilíndrica de grano fino TC-11EF (KENDO); las dimensiones de las preparaciones cavitarias fueron de $3 \mathrm{~mm}$ de largo, $4 \mathrm{~mm}$ de ancho y 2 $\mathrm{mm}$ de profundidad corroboradas por una sonda periodontal milimetrada carolina del norte (USA DELTA). Cada cavidad preparada fue lavada a chorro con jeringa triple y desinfectada con Gluconato de Clorhexidina al $2 \%$ (EUFAR) por 15 segundos.

Se procedió a dividir equitativamente la muestra en dos grupos, los cuales se diferencian como grupo A y grupo B; en los dientes del grupo A se utilizó el sistema adhesivo Adpter TM Single Bond 2 de la casa comercial 3M ESPE y en el grupo B se utilizó el sistema adhesivo Adhese Universal, Ivoclar Vivadent; todas las cavidades fueron restauradas con resina de microrelleno color A2 (FiltekTM Z250 XT, 3M ESPE), por medio de dos incrementos, que fueron fotopolimerizados por 20 segundos con lámpara de luz halógena (LITEXTM 680A). Para la terminación de todas las restauraciones se utilizó una fresa cilíndrica de grano fino TC-11EF (KENDO), posteriormente se pulieron con discos (PoliDisK) de grano mediano, fino y extra fino, y por último se utilizó pasta diamantada (Diamond excel FGM). Cada grupo fue conservado por separado en un baño maría (MEMMERT) con $100 \%$ de humedad a $37^{\circ} \mathrm{C}$ durante 24 horas.

Posterior al proceso mencionado cada muestra fue sometida a termociclado (300 ciclos con temperaturas de $5^{\circ} \mathrm{C}$ y $55^{\circ} \mathrm{C}$ por $60 \mathrm{seg}$ ), equivalente aproximado a 45 días de actividad en cavidad oral. ${ }^{11}$ Luego del termociclado se selló los ápices con cera rosada y se procedió a cubrir todas las superficies de los dientes con 3 capas de barniz de uñas transparente a un milímetro de la superficie restaurada. Toda la muestra se sumergió en solución acuosa de azul de metileno al $2 \%$ por 24 horas, luego cada diente fue lavado cuidadosamente bajo chorro de agua y frotado con un cepillo dental por 2 minutos.

Para observar la microfiltración presente los dientes fueron seccionados longitudinalmente a la restauración en sentido mesio-distal pasando por su centro, para ello, se utilizó un disco de diamante ultrafino (KENDO) montado en pieza de baja velocidad (NSK EX-203C) sin refrigeración, exponiendo la interfase diente-restauración; se utilizó un disco nuevo por cada quince dientes. Cada muestra fue evaluada por tres observadores previamente calibrados con microscopia estereoscópica (OLYMPUS SZ61 X 1,2).

Para clasificar el grado de microfiltración utilizamos la escala recomendada por Alavi y Kianimanesh ${ }^{5}$ investigadores que manifiestan: 


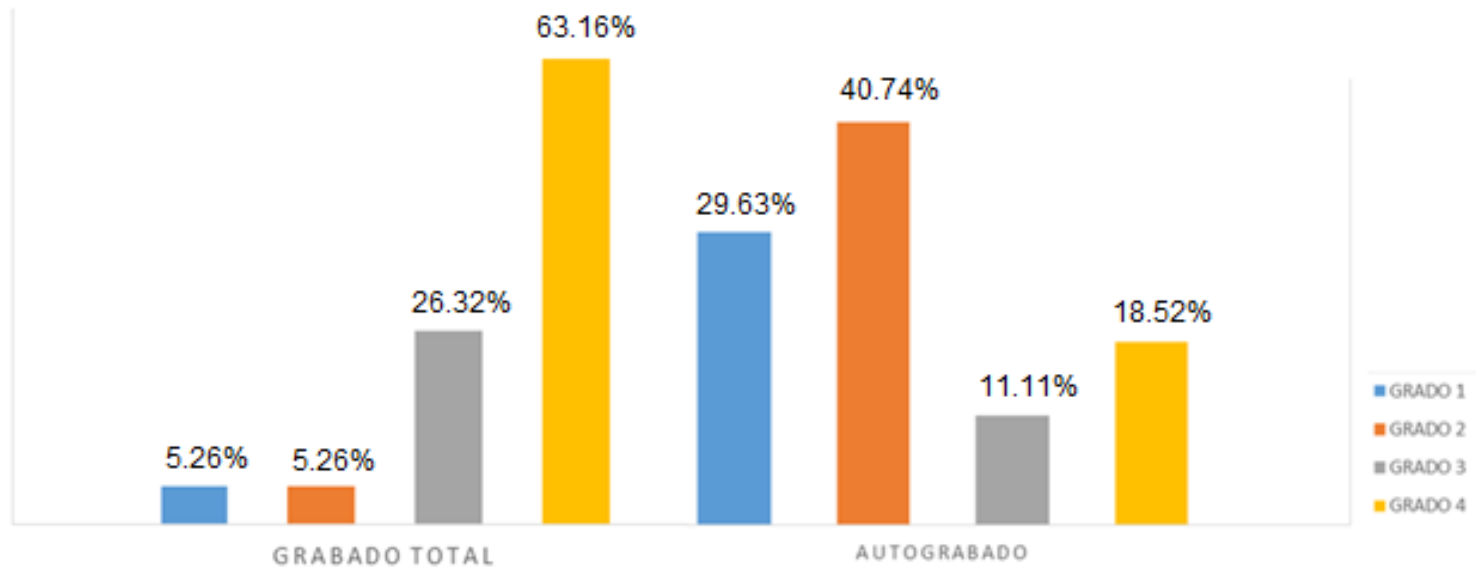

Grf. 1. Comparación de los grado de microfiltración entre el sistema adhesivo de grabado total y de autograbado.

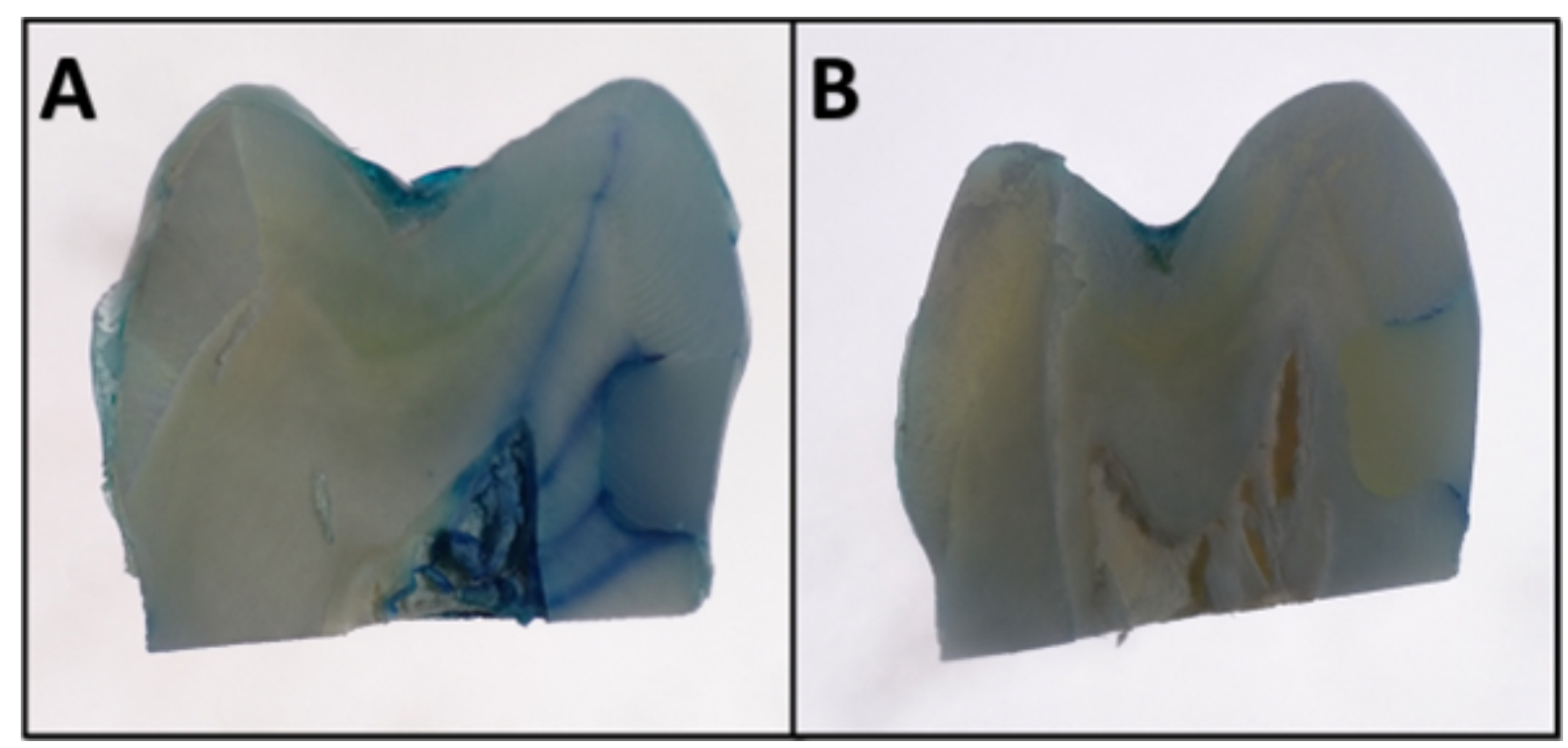

Fig. 1. Presencia de microfitracion marginal: A. "Grado 4" Sistema adhesivo de grabado total. B. "Grado 2" Sistema adhesivo de autograbado.

- $0=$ sin penetración de tinción.

- 1 = tinción penetrada $1 / 3$ superior de profundidad.

- 2 = tinción penetrada $1 / 3$ medio de profundidad.

- 3 = tinción penetrada $1 / 3$ apical a la pared axial de la cavidad.

- 4 = Penetración envolviendo la pared axial.

Para el análisis estadístico y comparación entre variables utilizamos la prueba del chi cuadrado $\mathrm{X}^{2}$.

\section{Resultados}

Del total de las piezas dentarias estudiadas el 71,21\% presentaron microfiltración marginal independientemente del sistema adhesivo empleado. El 84,84\% de especímenes del grupo A (sistema adhesivo de autograbado) presentaron microfiltración marginal; mientras que el $57,57 \%$ de especímenes del grupo B (sistema adhesivo de grabado total) presentaron dicha microfiltración. Existiendo diferencia estadísticamente significativa $(\mathrm{p}=0,03)$.

Del total de piezas dentarias que fueron acondicionadas con el sistema de grabado total con el mayor porcentaje $(63,16 \%)$ correspondió al grado 4, por el contrario al utilizar el sistema adhesivo de autograbado para acondicionar las piezas dentarias observamos la mayor proporción (40,74\%) de microfiltración con el grado 2 (Figura 1). Las diferencias observadas del grado de microfiltración al emplear los 
distintos tipos de sistemas adhesivos demuestran diferencia estadísticamente significativa ( $\mathrm{p}=0,0014)$. (Gráfico 1)

\section{Discusión}

El objetivo de nuestra investigación fue comparar el grado de microfiltración marginal utilizando sistemas adhesivos de grabado total y autograbado, por medio de infiltración de colorantes, ejecución de termociclado y observación estereoscópica.

Los resultados obtenidos sugieren que el acondicionamiento de las piezas dentarias mediate el sistema adhesivo de grabado total es superior al de autograbado, en términos de microfiltración marginal, con resultados similares a los publicados por Sánchez Ayala et. al $^{12}$ y Herrera Raya et al., ${ }^{13}$ quienes observaron diferencias significativas en los valores de microfiltración al acondicionar con el sistema adhesivo de autograbado y grabado total; ya que utilizaron sistemas adhesivos con una composición química similares a los empleados en el presente estudio.

Nicola et al, ${ }^{14}$ Kucukesmen y Sonmez ${ }^{15}$ y Gupta et al. ${ }^{16}$ determinaron que el sistema adhesivo de autograbado presentó mayor microfiltración que el sistema adhesivo de grabado total con un valor $\mathrm{p}$ de 0.03 a 0.05 ; coincidiendo con nuestra investigación dado que se realizó la técnica de termociclado. Por el contrario, en los estudios realizados por Pereira y Jordan, ${ }^{17}$ Alvarado et. al, ${ }^{18}$ Arias, Campos, Pimental $^{19}$ y Brackett et. al. ${ }^{20}$ demostraron que en las piezas dentarias acondicionadas con el sistema adhesivo de autograbado obtuvieron mayor grado de microfiltración que las piezas dentarias acondicionadas con el sistema adhesivo de grabado total; sin embargo en uno de estos estudios se empleo un número pequeño de especímenes, en otros se emplearon adhesivos de diferentes casas comerciales, o bien se incluyo un mayor numero de ciclos y en la totalidad de ellos no se sometieron al proceso de termociclado. ${ }^{21}$ Esto podría explicar las diferencias observadas entre los valores aquí presentados y los previamente reportados.

Diferentes estudios han demostrado que el solvente como parte de la composición química de los sistemas adhesivos, influye en la difusión del mismo. ${ }^{22}$ En nuestro estudio empleamos un sistema adhesivo de grabado total con solvente a base de alcohol, el mismo que permite una difusión mas efectiva en la superficie dentaria, influyendo en la estabilidad de la capa híbrida; y al ser previamente acondicionado con ácido ortofosfórico disuelve los minerales del sustrato dentario y expone las fibras colágenas obteniendo una adhesión química y micromecánica. ${ }^{23}$ Varias investigaciones sugieren que emplear sistemas adhesivos de autograbado con solventes a base de acetona se obtiene una difusión deficiente y que al no usar ácido ortofosfórico los minerales y fibras de colágeno se mantienen intactas obteniendo una adhesión únicamente química. ${ }^{24,25}$

Nuestra principal limitación fue utilizar un equipo de termociclado artesanal, manual y no automatizado, provocando errores y dificultades para su comparación con estudios realizados previamente.

Con esta información concluimos que los sistemas adhesivos de autograbado al incorporar el barrillo dentinario a la capa híbrida provocan una unión más estable entre el material restaurador y la superficie dentaria, provocando grados de microfiltración menores.

\section{Conclusiones}

- La microfiltración marginal observada fue alta independiente al sistema adhesivo utilizado.

- Existe diferencia estadísticamente significativa entre los sistemas adhesivos utilizados y la microfiltración marginal.

- El sistema adhesivo de grabado total obtuvo mayores grados de microfiltración en comparación al sistema adhesivo de autograbado.

Conflicto de intereses: Los autores declaran no tener conflicto de intereses.

\section{Referencias}

1 Mandri MN, Aguirre Grabre de Prieto A, Eugenia ZM. Sistemas adhesivos en Odontología Restauradora. Odontoestomatología. 2015 Noviembre; XVII(26).

2 Aguilar A, Barriga J, Terán R. Adhesivos de quinta y sexta generación. Revista Latinoamericana de Ortodoncia y Odontopediatría. 2015.

3 Carrillo S C. Dentina y adhesivos dentinarios. Conceptos actuales. ADM. 2006; LXIII(2).

4 Sánchez A, Farias A, Reis S, Carlos J, Mongruel O. Marginal microleakage of class $\mathrm{V}$ resin-based composite restorations bonded with six one-step self-etch systems. Brazilian Oral Research. 2013 Junio; XXVII(3).

5 Alavi A, Kianimanesh N. Microleakage of Direct and Indirect Composite Restorations with Three Dentin Bonding Agents. Operative Dentistry. 2002; 27(1).

6 Bravo S. Evaluación del Grado de Adhesión a la Dentina de Dos Tipos de Adhesivos de Uso Clínico Actual. Rev Dent Chile. 2011; 101(1).

7 Ramos Sánchez S, Calvo Ramírez N, Fierro Medina R. Adhesión Convencional En Dentina, Dificultades Y Avances En La Técnica. Rev Fac Odontol Univ Antioq. 2015; 26(2).

8 Vinay S, Shivanna V. Comparative evaluation of microleakage of fifth, sixth, and seventh generation dentin bonding agents: An in vitro study. Journal Conservative Dentistry. 2010 Jul-Sep; 13(3). 
9 Tabari M, Gharekhani S, Esmaeili B, Bejeh A, Mollaei 22 Ortega RA, Guerrero Ibarra J, Rivas LC. Microfiltración in M, Alimohammadi M, et al. Microleakage of Composite Resin Restorations Using a Type of Fifth and Two Types of Seventh Generations of Adhesive Systems: A Comparative 23 Study. JDMT. 16 Marzo; 5(1).

10 Lacouture Quintero L, Mendoza Duarte J, Rodrigo Rivera J. Efecto del ciclaje térmico en la fuerza y tipo de falla 2 adhesiva en esmalte humano. Ortodoncia. 2012.

11 Piedra Sarmiento LA, Ariza Salas MC, Guzmán Báez HJ, Zárate Cadena F. Comparison of debonding resistance and failure type of three orthodontic band cements. Revista 25 Facultad de Odontología Universidad de Antioquia. 2016; 28(1).

12 Sánchez Ayala A, Farias Neto A, Reis Vilanova L, Gomes J, Mongruel Gomes O. Marginal microleakage of class V resin- based composite restorations bonded with six onestep self-etch systems. Operative Dentistry. 2013 May-Jun; 27(3).

13 Herrera Raya S, Sánchez Sosme F, Reyes Missett GE, Vázquez Rodríguez EM, Guerrero Ibarra J. Microfiltración en restauraciones de resina realizadas con diferentes sistemas adhesivos estudio In Vitro. Revista Odontológica Latinoamerica. 2016; 8(2).

14 Nicola C, Sava S, Moldovana M, Prejmereana C, Romînub $\mathrm{M}$, Bondor C, et al. Effect of adhesive system on microleakage in class II direct composite restorations. Journal of optoelectronics and advanced materials. 2008 Abril; 10(4).

15 Küçükeşmen Ç, \& Sönmez H. Microleakage of class-v composite restorations with different bonding systems on fluorosed teeth. European Journal of Dentistry. 2008; 2.

16 Gupta A, Tavane P, Gupta PK, Tejolatha B, Lakhani AA, Tiwari R, et al. Evaluation of microleakage with total etch, self etch and universal adhesive systems in class $\mathrm{V}$ restorations: an in vitro study. Journal of Clinical and Diagnostic Research. 2017; 11(4).

17 Pereira Sánchez N, Jordán Barrios A. Microfiltración De Restauraciones Clase V De Resina Compuesta Colocadas con un adhesivo auto-acondicionante y un adhesivo de grabado total.. Odous Científica. 2007; 8(2).

18 Ordoñez GA, Astudillo AP, Lafebre Carrasco F. Análisis comparativo in vitro del grado de microfiltración entre un sistema adhesivo de quinta y séptima generación. Acta Odontológica Colombiana. 2016; 6(1).

19 Arias VG, Campos IT, Pimenta LAF. Microleakage study of three adhesive systems. Brazilian dental journal. 2004; 15(3).

20 Brackett WW, Haisch LD, Pearce MG, Brackett MG. Microleakage of Class $\mathrm{V}$ resin composite restorations placed with self-etching adhesives. The Journal of prosthetic dentistry. 2004; 91(1).

21 Asaka Y, Amano S, Rikuta A, Kurokawa H, Miyazaki M, Platt JA, et al. Influence of thermal cycling on dentin bond strengths of single-step self-etch adhesive systems. Operative dentistry. 2007; 32(1). vitro de tres sistemas adhesivos con diferentes solventes. Revista odontológica mexicana. 2012; 16(3).

23 Deliperi S, Bardwell DN, Wegley C. Restoration interface microleakage using one total-etch and three self-etch adhesives. Operative Dentistry. 2007; 32(2).

24 Geerts S, Bolette A, Seidel L, Guéders A. An in vitro evaluation of leakage of two etch and rinse and two selfetch adhesives after thermocycling. International journal of dentistry. 2012; 2012.

25 Bader, Espinoza. Análisis comparativo in vitro del grado de filtración marginal de restauraciones de resina compuesta realizadas utilizando el sistema adhesivo Adper ${ }^{\mathrm{TM}}$ Single bond 2 con grabado ácido y Single Bond ${ }^{\mathrm{TM}}$ Universal con y sin grabado ácido. Rev. Biomater. Sociedad científica Grupo Chileno de Materiales Dentales. 2015; 2(1).

Recibido: 29 de septiembre de 2020

Aceptado: 20 de noviembre de 2020 
